\title{
An Efficient Analytical Model of Coupled On-chip RLC Interconnects
}

\author{
Liang Yin and Lei He * \\ Electrical and Computer Engineering Department \\ University of Wisconsin, Madison, WI 53706
}

\begin{abstract}
In this paper, we present a new decoupled model for two coupled transmission lines with consideration of the inductive effect. It maps two coupled lines into two completely isolated lines with separated drivers and receivers, and has no loss of accuracy during the decoupling procedure. Further, we derive a closed-form time domain response for an isolated transmission line using a one-segment $R L C \Pi$ model. Combining the two models, we have an analytical time-domain solution to two coupled transmission lines. The model gives satisfied results for up to $5000 \mu m$-long lines when compared to SPICE simulation over an accurate distributed RLC circuit model, and can be used to model on-chip wires in the layout design, logic synthesis and high level design.
\end{abstract}

\section{Introduction}

For integrated circuits in the deep submicron (DSM) technology, interconnects play an important role in determining the performance and signal integrity $[1,2]$. An efficient onchip interconnect model is critical to interconnect optimization at high-level design, logic synthesis and physical design, as circuit simulation is overkill or not affordable at these design stages. Closed-form formulas are particularly efficient and effective for these design stages. Previous work includes formulas for the delay $[5,15,10]$, and formulas for the timedomain response $[6,11,12]$. The interconnect inductance is considered in $[10,6,12]$, but not in $[5,15,11]$.

All above methods consider only one isolated wire and ignore the coupling effect from neighboring wires. In DSM designs, the wire thickness is often larger than the wire width, and the spacing between adjacent wires is often smaller than the distance between adjacent metal layers. This makes the coupling capacitance between adjacent wires on the same layer larger than the ground capacitance to adjacent layers. The dominant coupling capacitance may cause delay variations and crosstalk. Further, the coupling inductance exists between both adjacent and non-adjacent wires. Ignoring the coupling inductance among a number of parallel wires may severely underestimate signal crosstalk [8].

* This research was partially supported by a grant from Intel, and used machines donated by SUN Microsystems. Address comments to lhe@ece.wisc.edu.

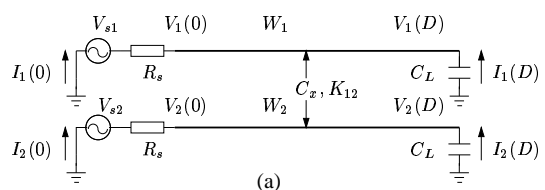

(a)

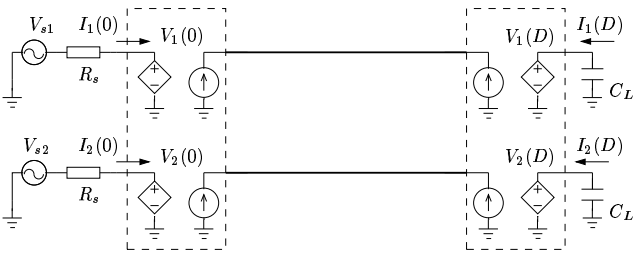

(b)

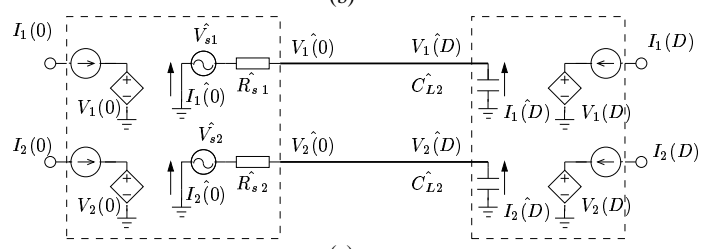

Figure 1: (a) Two identical coupled transmission lines. (b) The decoupled model in [7, 4], where the two lines are decoupled, but inputs $\left(V_{1}(0)\right.$ and $\left.V_{2}(0)\right)$ and outputs $\left(V_{1}(D)\right.$ and $\left.V_{2}(D)\right)$ of the decoupled lines are still coupled. (c) The new decoupled model proposed in this paper, where the two lines are decoupled, and the inputs $\left(\hat{V_{s 1}}\right.$ and $\left.\hat{V_{s 2}}\right)$ and outputs $\left(V_{1} \hat{(D}\right)$ and $\left.V_{2} \hat{(D)}\right)$ of the decoupled lines are also decoupled. Analytical formulas are developed to link $\hat{V_{s 1}}$ and $\hat{V_{s 2}}$ to the inputs of the original coupled lines, and to link $V_{1} \hat{(D)}$ and $V_{2} \hat{(D)}$ to the outputs of the original coupled lines.

Recently, a closed-form formula was developed for the coupling noise voltage in two identical RLC lines [16]. It is assumed that one wire is switching, and the other stays quiet. Further, both lines are open-ended without drivers and receivers. The formula is based on an approximate solution to the transfer function of the two coupled wires, and is valid only for the loosely coupled wires where the coupling noise in the switching wire can be ignored.

In this paper, we derive closed-form formulas for the time domain responses for two coupled RLC wires. The two wires are identical with identical drivers and receivers. We are able to consider an arbitrary combination of inputs and an arbitrary coupling strength. Our method is based on the modal analysis, which can decouple $n$ coupled lines into $n$ 
isolated lines [3, 4, 7, 17]. Therefore the coupled multi-line problem can be simplified as a number of isolated single-line problems. However, the decoupled relation was not developed for drivers and receivers, and numerical methods were used to solve the boundary problem due to drivers and receivers [7, 4]. The circuit model used in [4, 7] is shown in Figure 1(b) with the boundaries highlighted by boxes.

Our primary contribution in this paper is to show that the the decoupled relation holds for the boundaries for two identical RLC wires (see Figure 1(c)). Therefore, two coupled lines can be completely decoupled into two isolated lines, each with its own driver and receiver. We also propose a simple one segment $R L C \Pi$ model to approximate a single interconnect. The one-segment circuit model is justified by the fact that the on-chip wire (due to buffer insertion) is often shorter than the wave length at the signal operating frequency. Combining the decoupled model and the onesegment model, we have a closed-form time domain model for the two coupled wires.

The remainder of the paper is organized as follows: In section 2 , we introduce the modal analysis and the extension of the decoupled model to drivers and receivers. In section 3 , we present the time domain closed-form solution for the one-segment circuit model. We show experimental results in section 4 , and draw conclusions in section 5 .

\section{Decoupled model}

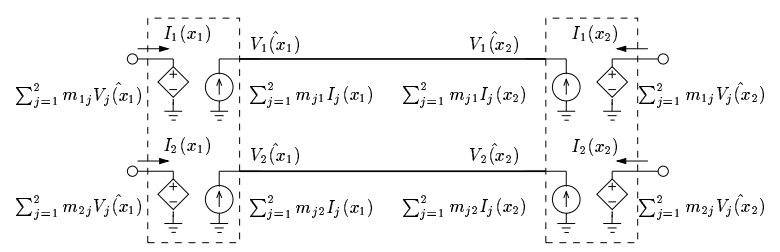

Figure 2: Decoupled equivalent transmission line model.

The two coupled transmission lines is described by

$$
-\frac{d}{d z}\left(\begin{array}{c}
\mathbf{V} \\
\mathbf{I}
\end{array}\right)=\left(\begin{array}{cc}
\mathbf{0} & \mathbf{Z} \\
\mathbf{Y} & \mathbf{0}
\end{array}\right)\left(\begin{array}{l}
\mathbf{V} \\
\mathbf{I}
\end{array}\right)
$$

where $\mathbf{V}\left(=\left(V_{1}(x) V_{2}(x)\right)^{T}\right), \mathbf{I}\left(=\left(I_{1}(x) I_{2}(x)\right)^{T}\right)$ are the line voltage and current vectors. $\mathbf{Z}(=\mathbf{R}+s \mathbf{L}), \mathbf{Y}(=\mathbf{G}+$ $s \mathbf{C})$ are unit impedance and conductance matrices for coupled transmission lines, respectively. Their dimensions are $2 \times 2$. Further, $0<x<D$ with $D$ being the length of the transmission line.

In the modal analysis, (1) can be described as

$$
-\frac{d}{d z}\left(\begin{array}{c}
\hat{\mathbf{V}} \\
\hat{\mathbf{I}}
\end{array}\right)=\left(\begin{array}{cc}
\mathbf{0} & \hat{\mathbf{Z}} \\
\hat{\mathbf{Y}} & \mathbf{0}
\end{array}\right)\left(\begin{array}{l}
\hat{\mathbf{V}} \\
\hat{\mathbf{I}}
\end{array}\right)
$$

with

$$
\left(\begin{array}{l}
\mathbf{V} \\
\mathbf{I}
\end{array}\right)=\left(\begin{array}{cc}
\mathbf{M} & \mathbf{0} \\
\mathbf{0} & \left(\mathbf{M}^{\mathbf{T}}\right)^{-\mathbf{1}}
\end{array}\right)\left(\begin{array}{l}
\hat{\mathbf{V}} \\
\hat{\mathbf{I}}
\end{array}\right)
$$

where $\hat{\mathbf{V}}\left(=\left(\hat{V}_{1}(x) \hat{V}_{2}(x)\right)^{T}\right), \hat{\mathbf{I}}\left(=\left(\hat{I}_{1}(x) \hat{I}_{2}(x)\right)^{T}\right)$ are voltage and current vectors for the decoupled lines. $\mathbf{M}$ is the voltage eigenvector matrix. The equivalent transmission line model is shown in Figure $2 . \hat{\mathbf{Z}}(=\hat{\mathbf{R}}+s \hat{\mathbf{L}}), \hat{\mathbf{Y}}(=\hat{\mathbf{G}}+s \hat{\mathbf{C}})$ are the unit impedance and conductance matrices for two decoupled transmission lines respectively. $\hat{\mathbf{Z}}$ and $\hat{\mathbf{Y}}$ are diagonal matrices with the dimensions of $2 \times 2$, and are given by

$$
\begin{aligned}
\hat{\mathbf{Z}} & =\mathbf{M}^{-\mathbf{1}} \mathbf{Z}\left(\mathbf{M}^{\mathbf{T}}\right)^{-\mathbf{1}} \\
\hat{\mathbf{Y}} & =\mathbf{M}^{\mathbf{T}} \mathbf{Y M}
\end{aligned}
$$

Based on the above equations, we can derive the expression of $\mathbf{M}$. If two lines are identical in terms of unit impedance, unit conductance and wire length, from (4) and (5)

$$
\mathbf{M}=\left(\begin{array}{ll}
m_{11} & m_{12} \\
m_{21} & m_{22}
\end{array}\right)=\left(\begin{array}{cc}
\sqrt{2} / 2 & \sqrt{2} / 2 \\
\sqrt{2} / 2 & -\sqrt{2} / 2
\end{array}\right)
$$

The above derivation are only valid for $0<x<D$ because the telegrapher equation (1) does not hold at the driver and receiver ends.

To extend the decoupled model to drivers and receivers, we model the driver by a voltage source with output resistance, and model the receiver by a load capacitance. As shown in Figure 1(a), we denote the driver voltages as $V_{s 1}$, $V_{s 2}$ for two lines, respectively. The driver resistance for both lines is $R_{s}$, and load capacitance is $C_{L}$. We have the following theorem:

Theorem The decoupled model (3) can be extended to the boundaries $(x=0, D)$ with

$$
\begin{aligned}
\left(\begin{array}{c}
\hat{V_{s 1}} \\
\hat{V_{s 2}}
\end{array}\right) & =\mathbf{M}^{-1}\left(\begin{array}{c}
V_{s 1} \\
V_{s 2}
\end{array}\right) \\
\hat{R_{s 1}}=\hat{R_{s 2}} & =R_{s} \\
\hat{C_{L 1}}=\hat{C_{L 2}} & =C_{L}
\end{aligned}
$$

where $\hat{V_{s 1}}$ and $\hat{V_{s 2}}$ are the driver voltages, $\hat{R}_{s}^{1}$ and $\hat{R}_{s}^{2}$ are the driver resistances, and $\hat{C}_{L}^{1}$ and $\hat{C}_{L}^{2}$ and the load capacitances, all for the two decoupled lines, respectively. The decoupled circuit with driver/receiver is shown in Figure 1(c).

Proof: Let's consider the driver ends $(x=0)$ first. By circuit analysis, we can derive the boundary conditions of two networks

$$
\begin{aligned}
& \left(\begin{array}{l}
V_{1}(0) \\
V_{2}(0)
\end{array}\right)=\left(\begin{array}{c}
V_{s 1}-I_{1}(0) R_{s} \\
V_{s 2}-I_{2}(0) R_{s}
\end{array}\right) \\
& \left(\begin{array}{c}
V_{1} \hat{(0)} \\
V_{2} \hat{(0)}
\end{array}\right)=\left(\begin{array}{c}
\hat{V_{s 1}}-I_{1} \hat{(0)} R_{s} \\
\hat{V_{s 2}}-I_{2} \hat{(0)} R_{s}
\end{array}\right)
\end{aligned}
$$


If the relation (3) holds at the driver ends, from (10) and (11), we have

$$
\left(\begin{array}{c}
V_{s 1}-I_{1}(0) R_{s} \\
V_{s 2}-I_{2}(0) R_{s}
\end{array}\right)=\mathbf{M}\left(\begin{array}{c}
\hat{V_{s 1}}-\hat{I_{1}} \hat{(0)} \hat{R_{s 1}} \\
\hat{V_{s 2}}-I_{1} \hat{(0)} \hat{R_{s 2}}
\end{array}\right)
$$

Because

$$
\left(\begin{array}{c}
V_{s 1} \\
V_{s 2}
\end{array}\right)=\mathbf{M}\left(\begin{array}{c}
\hat{V_{s 1}} \\
\hat{V_{s 2}}
\end{array}\right)
$$

and

$$
\left(\begin{array}{c}
I_{1}(0) R_{s} \\
I_{2}(0) R_{s}
\end{array}\right)=\mathbf{M}\left(\begin{array}{c}
\hat{I_{1}} \hat{(0)} \hat{R_{s 1}} \\
I_{2} \hat{(0)} \hat{R_{s 2}}
\end{array}\right)
$$

From (3), (6) and (13), we have

$$
\begin{aligned}
& I_{1}(0) R_{s}=\frac{1}{2} I_{1}(0)\left(\hat{R_{s 1}}+\hat{R_{s 2}}\right)+\frac{1}{2} I_{2}(0)\left(\hat{R_{s 1}}-\hat{R_{s 2}}\right) \\
& I_{2}(0) R_{s}=\frac{1}{2} I_{1}(0)\left(\hat{R_{s 1}}-\hat{R_{s 2}}\right)+\frac{1}{2} I_{2}(0)\left(\hat{R_{s 1}}+\hat{R_{s 2}}\right)
\end{aligned}
$$

Then $\hat{R_{s 1}}=\hat{R_{s 2}}=R_{s}$. Similarly we can prove $C_{L 1}=$ $C_{L 2}=C_{L}$ at the receiver ends.

We use Figures 3, 4 and 5 to illustrate our decoupled model. We apply exponential signals switching at opposite directions to the two coupled lines, and compare the spice simulation results over the coupled lines with the results derived from the decoupled model. The response of each isolated (i.e., decoupled) line is also computed by spice simulation. Further, the case where one line is switching and the other line is quiet will be presented in section 4 .

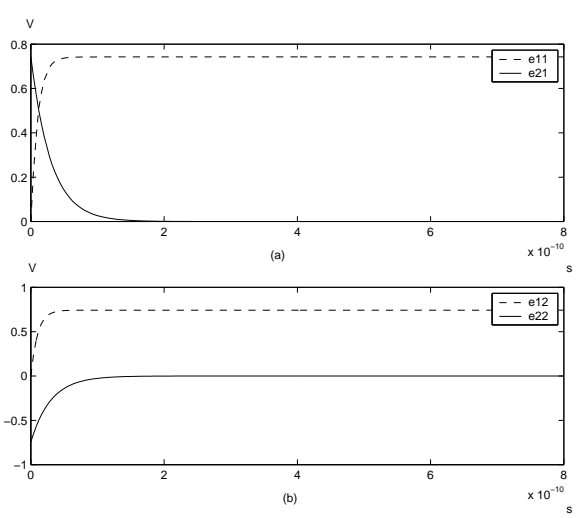

Figure 3: Input components $\left(e_{i j}\right)$ to decoupled lines: (a) decoupled line 1 , and (b) decoupled line 2. Based on equation (12), inputs to isolated lines are $\hat{V}_{s 1}=e_{11}+e_{21}, \hat{V_{s 2}}=e_{12}+e_{22}$, where $e_{i j}=\left(\mathbf{M}^{-1}\right)_{i j} V_{s j}$ with $i, j=1,2$. Parameters: $D=3000 \mu$, $R=12.94 \Omega, L=4.70 n H, C=110 f F, C_{x}=96.5 f F$ and $K_{12}=0.895$. $V_{s 1}$ and $V_{s 2}$ are both exponential inputs where the rising constant is $10 p s$ for $V_{s 1}$, and is $30 p s$ for $V_{s 2}$.

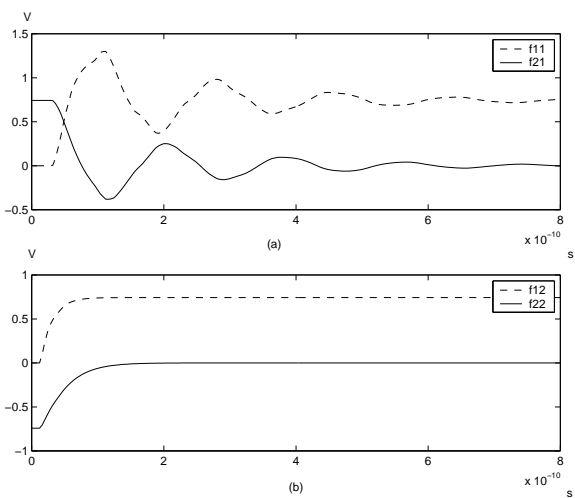

Figure 4: Output components at the receiver ends of two decoupled lines. $f_{i j}$ is SPICE simulation output of single transmission line (decoupled line) where the input is $e_{i j}$. (a) At line 1 where $V_{1} \hat{(D)}=f_{11}+f 21$. (b) At line 2 where $V_{2} \hat{(D)}=f_{12}+f_{22}$. Here $D=3000 \mu$.

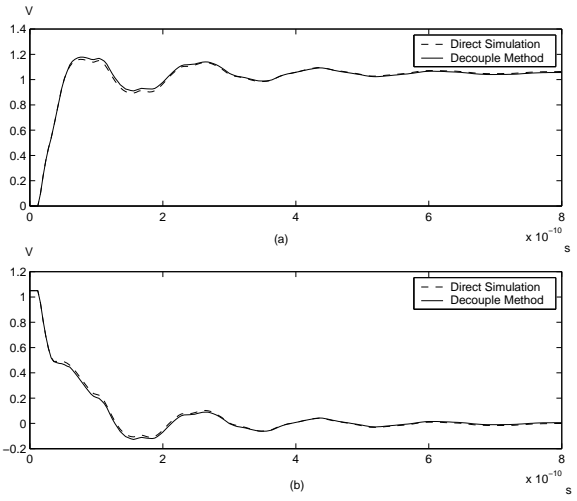

Figure 5: Comparison of direct simulation and the result from decoupling method by coupling four components from Figure 4. (a) Comparison at the output end of line 1. (b) Comparison at the output end of line 2. Obviously They are almost the same. 


\section{Solution to one line}

Using the modal analysis and driver/receiver mapping, we can simplify the coupled line problem into the single-line problem. Because the open-ended transmission line is assumed in [6] and the inductance is not considered in [11], the single line solutions in $[6,11]$ are not applicable to our case. As buffers are often used in DSM designs to break long wires into short wires, on-chip wires are often shorter than the wave length at the signal operating frequency. Therefore, the distributed effect often is not distinct for on-chip wires, and we can model an on-chip interconnect as a one-segment $R L C \Pi$ circuit. As shown as in Figure $6, R, L, C$ are the resistance, inductance and capacitance of the line, respectively. $R_{s}$ is the driver resistance, and $C_{L}$ is the load capacitance. $C_{1}=\frac{1}{2} C$ and $C_{2}=\frac{1}{2} C+C_{L}$. The transfer function of the circuit is

$$
H=\frac{1}{1+a_{1} s+a_{2} s^{2}+a_{3} s^{3}}
$$

where

$$
\begin{aligned}
& a_{1}=\left(R+R_{s}\right) C_{2}+R_{s} C_{1} \\
& a_{2}=\left(R R_{s} C_{1 s}+L\right) C_{2} \\
& a_{3}=R_{s} C_{1} C_{2} L
\end{aligned}
$$

The transfer function (14) can be expressed in rational form

$$
H=\frac{k_{1}}{s-p_{1}}+\frac{k_{2}}{s-p_{2}}+\frac{k_{3}}{s-p_{3}}
$$

where

$$
\begin{aligned}
p_{1} & =\frac{1}{6} \frac{t_{2}}{a_{3}}-\frac{2}{3} \frac{t_{3}}{a_{3} t_{2}}-\frac{1}{3} \frac{a_{2}}{a_{3}} \\
p_{2} & =-\frac{1}{12} \frac{t_{2}}{a_{3}}+\frac{1}{3} \frac{t_{3}}{a_{3} t_{2}}-\frac{1}{3} \frac{a_{2}}{a_{3}} \\
& +I \frac{\sqrt{3}}{12}\left(\frac{t_{2}}{a_{3}}+\frac{4 t_{3}}{a_{3} t_{2}}\right) \\
p_{3} & =-\frac{1}{12} \frac{t_{2}}{a_{3}}+\frac{1}{3} \frac{t_{3}}{a_{3} t_{2}}-\frac{1}{3} \frac{a_{2}}{a_{3}} \\
& -I \frac{\sqrt{3}}{12}\left(\frac{t_{2}}{a_{3}}+\frac{4 t_{3}}{a_{3} t_{2}}\right)
\end{aligned}
$$

and

$$
\begin{aligned}
t_{1} & =4 a_{1}^{3} a_{3}-a_{1}^{2} a_{2}^{2}-18 a_{1} a_{2} a_{3}+27 a_{3}^{2}+4 a_{2}^{3} \\
t_{2} & =\sqrt[3]{36 a_{1} a_{2} a_{3}-108 a_{3}^{2}-8 a_{2}^{3}+12 \sqrt{3 t_{1}} a_{3}} \\
t_{3} & =3 a_{1} a_{3}-a_{2}^{2}
\end{aligned}
$$

Further, $p_{1}$ is a real number, and $p_{2}$ and $p_{3}$ are complex values, i.e., $p_{2}=p_{m}+I p_{n}$ and $p_{3}=p_{m}-I p_{n}$. Similarly, $k_{1}$ is real, and $k_{2}$ and $k_{3}$ can be expressed as $k_{2}=k_{c}+I k_{d}$ and $k_{3}=k_{c}-I k_{d}$, respectively. By setting (14)=(18), $k_{1}, k_{2}$ and $k_{3}$ can be calculated.

$$
\begin{aligned}
k_{1} & =\frac{1}{a_{3}\left(p_{1}^{2}+p_{m}^{2}+p_{n}^{2}-2 p_{1} p_{m}\right)} \\
k_{c} & =-\frac{1}{2 a_{3}\left(p_{1}^{2}+p_{m}^{2}+p_{n}^{2}-2 p_{1} p_{m}\right)} \\
k_{d} & =\frac{p_{1}-p_{m}}{2 p_{n} a_{3}\left(p_{1}^{2}+p_{m}^{2}+p_{n}^{2}-2 p_{1} p_{m}\right)} \\
k_{2} & =k_{c}+I k_{d} \\
k_{3} & =k_{c}-I k_{d} \\
\mathrm{~N}_{\text {in }} & \mathrm{N}_{\mathrm{C}_{1}}
\end{aligned}
$$

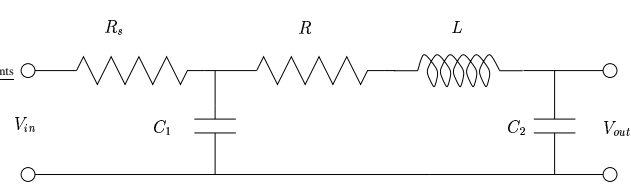

Figure 6: One-segment $R L C \Pi$ model

The $s$-domain expression for exponential rising input can be expressed as

$$
V i n_{s}=V_{0}\left(\frac{1}{s}-\frac{1}{s+t_{r}^{-1}}\right)
$$

where $V_{0}$ is the amplitude and $t_{r}$ is the rising time constant. Combine (27) and (18), the output is

$$
\begin{aligned}
\text { Vout }_{s} & =\text { HVin }_{s} \\
& =\sum_{1 \leq i \leq 3} V_{0}\left(\frac{k_{i}}{s-p_{i}}\right)\left(\frac{1}{s}-\frac{1}{s+t_{r}^{-1}}\right) \\
& =\sum_{1 \leq i \leq 3} V_{0} k_{i}\left[\frac{t_{r}^{-1}}{p_{i}\left(p_{i}+t_{r}^{-1}\right)} \frac{1}{s-p_{i}}\right. \\
& \left.-\frac{1}{p_{i} s}-\frac{1}{\left(p_{i}+t_{r}^{-1}\right)\left(s+t_{r}^{-1}\right)}\right]
\end{aligned}
$$

Therefore the time domain response of output signal is

$$
\begin{aligned}
\text { Vout }_{t} & =\sum_{1 \leq i \leq 3} V_{0} k_{i}\left[\frac{t_{r}^{-1}}{p_{i}\left(p_{i}+t_{r}^{-1}\right)} e^{p_{i} t}\right. \\
& \left.-\frac{1}{p_{i}}-\frac{1}{p_{i}+t_{r}^{-1}} e^{-t_{r}^{-1} t}\right]
\end{aligned}
$$

\section{Experimental Results}

Combining the one line closed-form solution (29) and our new decoupled model, we achieve a closed-form solution to two coupled lines. In this section, we compare our closedform solutions to spice simulation results over a full RLC model [9]. In the full model, we use $30 R L C$ segments to approximate a long wire. We consider the mutual inductance 

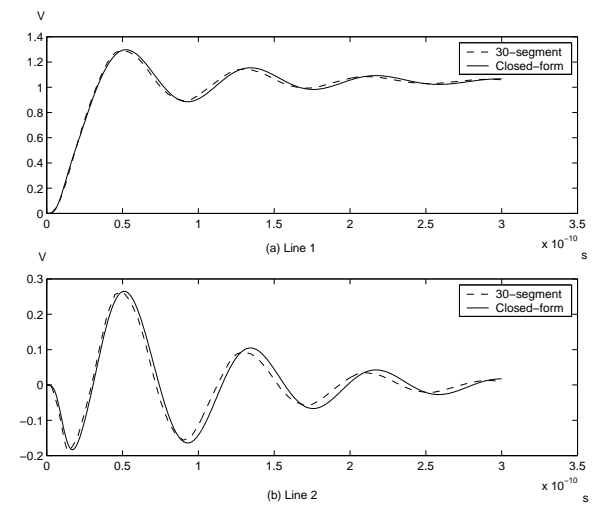

Figure 7: Comparison of closed-form solution with full model spice simulation results. Parameters: $D=1000 \mu, R=4.31 \Omega$, $L=1.35 n H, C=36.7 f F, C_{x}=32.2 f F$ and $K_{12}=0.88$ where $K_{12}$ is the coupling coefficient between two lines.

between any two segments in order to achieve an highly accurate model.

We test three difference cases, where the wire length are $1000 \mu, 3000 \mu$ and $5000 \mu$, respectively. The line width and the distance between two lines are both $2 \mu$. The comparisons are shown in Figures 7, 8 and 9. For all cases, one exponential rising input with rising $T_{r}=10 p s$ and amplitude $V_{0}=1.05 \mathrm{~V}$ is applied to line 1 while line 2 stays quiet. In all figures, $(a)$ represents the output signal at the output end of the aggressor, and $(b)$ for the output of the victim line.
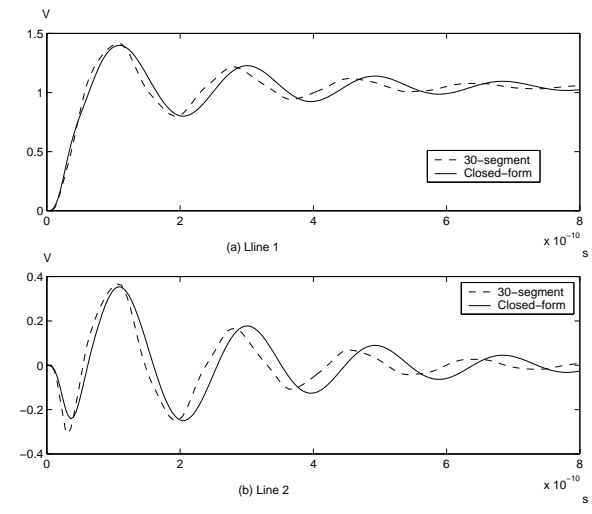

Figure 8: Comparison of closed-form solution with full model spice simulation results. Parameters: $D=3000 \mu, R=12.94 \Omega$, $L=4.70 n H, C=110 f F, C_{x}=96.5 f F$ and $K_{12}=0.895$.

For wires with length of $1000 \mu$ (see Figure 7), the waveform given by closed-form formulas is almost identical to the full model simulation results. It verifies our previous observation that when an interconnect is short enough compared to the wavelength of the signal operating frequency, the distributed transmission line model is not necessary for interconnect analysis.

The solution to the $5000 \mu$-long wires has the worst ac-
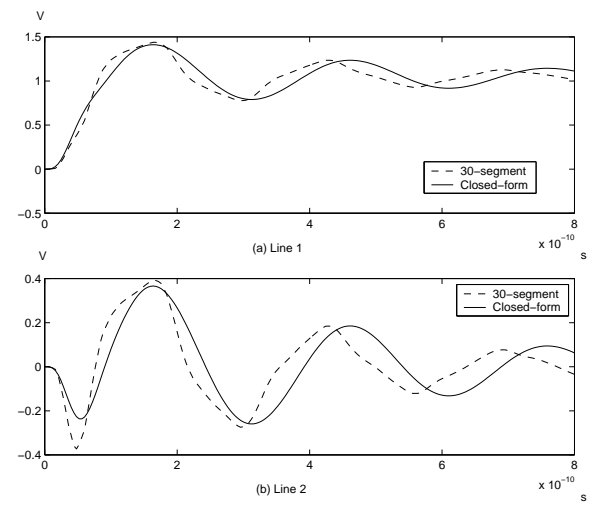

Figure 9: Comparison of closed-form solution with full model spice simulation results. Parameters: $D=5000 \mu, R=21.57 \Omega$, $L=8.35 n H, C=183 f F, C_{x}=160 f F$ and $K_{12}=0.90$.

curacy (Figure 9), while the accuracy for $3000 \mu$-long wires (Figure 8) is between those for $1000 \mu$ - and $5000 \mu$-long wires. In the following, we measure the error compared to SPICE simulation over the full RLC model for the first peaks at receiver ends. For the switching aggressor (Figure 9(a)), both the peak voltage and the $50 \%$ delay differ by $3 \%$. For the quiet victim (Figure 9(b)), the peak voltage differs by $5 \%$, and the $50 \%$ delay differs by $18 \%$. Clearly, the distributed effect becomes stronger with the increase of the wire length. Nevertheless, the closed-form solution to the $5000 \mu$ long wires is still good enough to guide interconnect optimization.

In real designs, $5000 \mu$ is often larger than the allowed maximum wire length with consideration of buffer insertion. Because the noise for the $5000 \mu$-long wires is more than $20 \%$ of supply voltage (Figure 9), such long wires are often broken into several short wires by buffers for performance and signal integrity purposes $[1,2]$. Therefore the closedform solution of the one-segment $R L C \Pi$ model is sufficient for interconnect analysis in most real designs.

\section{Conclusion}

In this paper, we present a new decoupled analysis model for two coupled RLC wires. It maps two coupled transmission lines into two isolated lines with separated driver/receiver. Furthermore, we use a one-segment $R L C \Pi$ circuit to model a single isolated wire, and then derive a closed-form time domain response for exponential rising inputs. Combining the decoupled model and the one-segment $R L C \Pi$ model, we have a closed-form time domain model for the two coupled lines. This model can be used to guide the layout design, logic synthesis and high-level design. Our future work will extend the method to multiple nets, and to non-identical drivers and receivers. 


\section{References}

[1] J. Cong, L. He, C. K. Koh, and P. H. Madden, "Performance Optimization of VLSI Interconnect Layout," Integration, Vol. 21, pp. 1 - 94, 1996.

[2] J. Cong, L. He, K. Y. Khoo, C. K. Koh, and Z. Pan, "Interconnect Design for Deep Submicron ICs," ICCAD, pp. $478-485,1997$.

[3] Tom Dhaene and Daniel De Zutter, "Selection of Lumped Element Models for Coupled Lossy Transmission Lines", IEEE Transactions on ComputerAided Design, Vol. 11, No. 7, pp. 805 - 815, July, 1992.

[4] A. R. Djordjevic, T. K. Sarkar and R. F. Harrinton, "Time Domain Response of Multiconductir Transmission Lines", Proc. IEEE, vol. 75, pp. $743-764$, June 1987.

[5] E. Elmore, "The Transient Response of Damped Linear Network with Particular Regard to Wideband Amplifiers", Journal of Applied Physics, pp. 55-63, Jan, 1948.

[6] Y. Gao and D.F. Wong, "Shaping a VLSI Wire to Minimize Delay Using Transmission Line Model", Design Digest of Technical Papers, ACM, New York, pp. 611 - 616, 1998.

[7] K. D. Granzow "Digital Transmission Lines", OXford University Press, 1998

[8] L. He and K. M. Lepak, "Simultaneous shielding insertion and net ordering for capacitive and inductive coupling minimization", ACM/IEEE International Symposium on Physical Design, 2000.

[9] L. He and M. Xu, "Characteristics and Modeling for On-Chip Inductive Coupling”, Univ. of Wisconsin-Madison Technique Report, ECE-00-01. http://eda.ece.wisc.edu/publications.html

[10] Y. I. Ismail, E. G. Friedman and J. L. Neves, "Equivalent Elmore Delay for RLC Trees", IEEE Transactions on Computer-Aided Design of Integrated Circuits and Systems, Vol. 19, No. 1, Jan 2000.

[11] A.B. kahng and S. Muddu, "Optimal Equivalent Circuit for Interconnect Delay Calculations Using Moments", European Design Automation Conference, 1994.

[12] B. Krauter, R. Gupta, J. Willis, and L. T. Pileggi, "Transmission Line Synthesis", Design Automation Conference, 1995
[13] Tuyen V. Nguyen, "Efficient Simulation of Lossy and Dispersive Transmission Lines" Proceedings of European Design Automation Conference pp. $622-$ 627,1994

[14] J. Rabaey, "Digital Integrated Circuits: A Design Perspective", Prentice Hall, 1996.

[15] T. Sakuri, "Closed-form expressions for interconnection delay, coupling, and crosstalk in VLSI's", IEEE Transactions on Electron Devices, vol. 40, pp. 118 - 124, Jan. 1993.

[16] Kevin T. Tang and Eby G. Friedman, "Interconnect Coupling Noises in CMOS VLSI Circuits", ISPD, pp. $48-53,1999$.

[17] V. K. Tripathi and J. B. Rettig, "A Spice Model for Multiple Coupled Microstrips and Other Transmission Lines", IEEE Transactions on Microwave Theory and Techniques, Vol. MTT-33, No. 12, pp. 1513 - 1518, Dec 1985. 